\title{
Prevalence of Chronic Kidney Disease and Associated Factors in Senegalese Populations: A Community-Based Study in Saint-Louis
}

\author{
Sidy Mohamed Seck ${ }^{1, *}$; Dominique Doupa ${ }^{2}$; Lamine Gueye $^{3}$; Charles Abdou Dia ${ }^{4}$ \\ ${ }^{1}$ Department of Internal Medicine and Nephrology, Faculty of Health Sciences, University Gaston Berger, Saint-Louis, Senegal \\ 2 Department of Biochemistry, Faculty of Health Sciences, University Gaston Berger, Saint-Louis, Senegal \\ ${ }^{3}$ Department of Neurology, Faculty of Health Sciences, University Gaston Berger, Saint-Louis, Senegal \\ ${ }^{4}$ Department of Neurology, Faculty of Health Sciences, University Gaston Berger, Saint-Louis,
${ }^{2}$ Menerane Outpatient Clinic, District Hospital of Saint-Louis, Saint-Louis, Senegal \\ ${ }^{*}$ Corresponding author: Sidy Mohamed Seck, Department of Internal Medicine and Nephrology, Faculty of Health Sciences, University Gaston Berger, Saint-Louis, Senegal. Tel/Fax: \\ +221-339619974, E-mail: sidymseck@gmail.com
}

Received: March 21, 2014; Revised: April 24, 2014; Accepted: April 27, 2014

\begin{abstract}
Background: Chronic kidney disease (CKD) is an emerging worldwide epidemic but littledata concerning African populations are available.

Objectives: We aimed to assess prevalence of CKD in adult populations of Saint-Louis, northern Senegal.

Patients and Methods: In a population-based survey between January and May 2012, we included 1037 adults $\geq 18$ years of age who resided in Saint-Louis. Socio-demographic, clinical, and biologic data were collected during household visits. Serum creatinine was measured by Jaffé method. We estimated glomerular filtration rate (eGFR) using the four-variable Modification of Diet in Renal Disease (MDRD) equation and CKD was defined by eGFR $<60 \mathrm{~mL} / \mathrm{min} / 1.73 \mathrm{~m}^{2}$ and/or albuminuria $>1 \mathrm{~g} / \mathrm{L}$. A multivariate logistic regression was performed to identify factors associated with CKD.

Results: The mean of participants' age was $47.9 \pm 16.9$ years (range, 18-87) and sex ratio (male to female) was 0.52. Majority of participants lived in urban areas (55.3\%) and had school education (65.6\%). Hypertension, diabetes, and obesity were present respectively in $39.1 \%, 12.7 \%$, and $23.4 \%$ of participants. Overall CKD prevalence was $4.9 \%(95 \% \mathrm{CI}, 3.5-6.2)$ with eGFR $<30 \mathrm{~mL} / \mathrm{min} / 1.73 \mathrm{~m}^{2}$ in $0.9 \%$. Albuminuria $>1 \mathrm{~g} / \mathrm{L}$ was found in $3.5 \%$ of patients. CKD was significantly more frequent among hypertensive patients in comparison with normotensive ones. Risk factors associated with CKD were hypertension ( $12 \%$ of risk excess) and age ( $3 \%$ of risk excess).

Conclusions: CKD is frequent in adult population living in NorthernSenegal. Main associated factors are hypertension and age. Prevention strategies are urgently needed to raise public awareness and promote early CKD detection and treatment in both urban and rural areas.
\end{abstract}

Keywords:Kidney Disease; Epidemiology; Population; Senegal

\section{Background}

Chronic kidney disease (CKD) represents one of the greatest public health challenges in the 21st century and is associated with considerable cardiovascular morbidity and mortality $(1,2)$. It has a major effect on healthcare costs and world productivity, particularly in low-income countries where the young people are the most afflicted population (3). Early detection of CKD along with an adequate management of patients is the best strategy to fight this disease. In Africa, although we assist with raising awareness of health authorities about CKD burden, developing prevention and management programs are still difficult due to the scarcity of epidemiologic data at population level $(3,4)$. Most of the current data come from nephrology departments in specialized hospitals in big cities and there is very little information concerning rural populations (5).

\section{Objectives}

This study aimed to assess prevalence of CKD in urban and rural areas of Saint-Louis, northern region of Senegal.

\section{Patients and Methods}

\subsection{Study Design}

We performed a community-based cross-sectional study in Saint-Louis (northern region of Senegal). All individuals who aged $\geq 18$ years and were residing in Saint-Louis for at least three months were eligible to participate in the study.

\subsection{Sampling Procedure}

A two-stage cluster sampling method was used to select a representative sample of adults living in urban and rural areas of Saint-Louis. We firstly selected 17 localities as clusters (nine urban areas and eight rural areas). Then we randomly took a number of households proportional to population size of each locality (data available from the National Agency of Statistics and Demography). From each household, a maximum of two participants were recruited. Considering aerror of 0.05 and power of $80 \%$, the required sample size was 855 individuals and we added a $20 \%$ attrition rate to get a final sample of 1026 participants.

Copyright (C) 2014, Nephrology and Urology Research Center; Published by Kowsar Corp. This is an open-access article distributed under the terms of the Creative Commons Attribution License, which permits unrestricted use, distribution, and reproduction in any medium, provided the original work is properly cited. 


\subsection{Data Collection}

Data were collected onsite during house-to-house visits that were conducted between 7 A.M. and 12 A.M. or, when patients did not live far away from this facility, at the nearest health center. A modified version of the WHO STEP wise questionnaire was pretested and validated. Researchers were assisted by medical students, trained nurse practitioners, and community health workers to fill the data collection form to document the socio-demographic status (age, sex, marital status, education, profession, and education), personal and family health history (regarding particularly hypertension, diabetes, stroke, heart and kidney disease), and lifestyle (nutritional habits, physical activity, smoking, and alcohol consumption) of each participant. History of nephrotoxic medications (nonsteroidal anti-inflammatory drugs and traditional herbs) was also recorded. Anthropometric measurements (weight, height, and waist and hip circumference) were performed using standard methods and calibrated devices. Serum total cholesterol, LDL, HDL, and triglycerides were measured with colorimetric method. Obesity was defined using International Diabetes Foundation cutoffs (6). Blood pressure was measured twice at five minutes intervals by a semiautomatic sphygmomanometer and the mean of the two readings was calculated. If the difference between the readings was greater than 10 $\mathrm{mmHg}$, a third measurement was performed.

Hypertension was defined as a systolic blood pressure $\geq 140 \mathrm{mmHg}$, diastolic blood pressure $\geq 90 \mathrm{mmHg}$, any prescription of antihypertensive medication in the past two weeks, or any self-reported history of hypertension (7). Fasting blood glucose (FBG) was measured with glucose oxidase method. Diabetes was defined as FBG $\geq 1.26 \mathrm{mg} /$ $\mathrm{dL}$, prescription of hypoglycemic agents despite normal fasting plasma glucose values, or any self-reported history of diabetes (6). Physical inactivity was defined as $<30 \mathrm{~min}$ - utes of moderate activity per week or $<20$ minutes of vigorous activity three times a week, or the equivalent. Serum creatinine was measured with Jaffé kinetic method and glomerular filtration rate (GFR) was estimated with the Modification of Diet in Renal Disease (MDRD) equation (8). Urinary albumin excretion (UAE) was first screened using strips and positive cases were confirmed by a quantitative dosage in the 24-hour urine output. CKD was defined according to National Kidney Foundation classification (9).

\subsection{Ethical Issues}

The National Committee for Ethics in Health Research approved the study. An informed consent form had to be signed by participants to obtain their approval before data collection. All participants were personally informed of their screening results and those with abnormal values were referred to a specialist for further exploration and treatment.

\subsection{Statistical Analysis}

Statistical analyses were performed with STATA 12.0 (StataCorp, TX, USA). Continuous variables were presented as mean \pm standard deviation (SD) and categorical variables as frequency and percentage. Comparison of proportions and means were done using Pearson's Chisquare test or Student's t-test as appropriated. Multivariate regression analysis was used to identify clinical and biological parameters associated with CKD. A P value $\leq 0.05$ was considered as statistically significant.

\section{Results}

A total of 1047 individuals were interviewed and 1037 had a complete questionnaire. One individual did not have serum creatinine and were kept in the study (response rate, $99 \%$ ). Overall, $53 \%$ of them lived in urban areas and $60 \%$ were female. Table 1 presents demographic, clinical,

\begin{tabular}{|c|c|c|c|c|}
\hline & All Participants, $(n=1037)$ & Urban Areas, $(n=578)$ & Rural Areas, $(n=459)$ & PValue \\
\hline Age, $y$ & $48.0 \pm 16.9$ (range, $18-87)$ & $51.6 \pm 15.7$ & $43.5 \pm 17.2$ & 0.001 \\
\hline Age Group, y & & & & 0.001 \\
\hline $18-34$ & $25.6 \%$ & $16.0 \%$ & $37.8 \%$ & \\
\hline $35-49$ & $25.3 \%$ & $26.1 \%$ & $24.2 \%$ & \\
\hline $50-60$ & $23.6 \%$ & $28.0 \%$ & $18.1 \%$ & \\
\hline$>60$ & $25.5 \%$ & $29.9 \%$ & $19.9 \%$ & \\
\hline School Education & $60.7 \%$ & $63.4 \%$ & $55.6 \%$ & 0.030 \\
\hline Familial History of Renal Disease & $10.4 \%$ & $5.4 \%$ & $15.0 \%$ & 0.003 \\
\hline Physical Inactivity & $58.1 \%$ & $55.3 \%$ & $61.7 \%$ & 0.047 \\
\hline Hypertension & $39.1 \%$ & $43.3 \%$ & $33.8 \%$ & 0.002 \\
\hline Diabetes (FBG $\geq 1.26 \mathrm{~g} / \mathrm{L}$ ) & $12.7 \%$ & $14.6 \%$ & $10.3 \%$ & 0.038 \\
\hline Obesity (BMI $\geq 30 \mathrm{~kg} / \mathrm{m}^{2}$ ) & $23.4 \%$ & $33.8 \%$ & $10.2 \%$ & $<0.001$ \\
\hline Cholesterol, g/L & $2.18 \pm 0.49$ & $2.25 \pm 0.54$ & $2.10 \pm 0.44$ & $<0.001$ \\
\hline eGFR, $\mathrm{mL} / \mathrm{min}$ & $90.6 \pm 23.8$ & $89.7 \pm 22.8$ & $91.7 \pm 24.9$ & 0.203 \\
\hline Albuminuria $>1 \mathrm{~g} / \mathrm{L}$ & $5.3 \%$ & $4.3 \%$ & $6.6 \%$ & 0.178 \\
\hline
\end{tabular}


Seck SM et al.

and biologic characteristics of participants according to their living areas. Compared to rural population, participants from urban cities were older, more educated, more active, and more likely to be women. Approximately, 10\% of participants reported a relative with a history of renal disease. Cardiovascular risk factors such as hypertension, diabetes, and obesity were frequent among the whole population with a significantly higher prevalence in individuals living in urban areas (Table 1).

Overall adjusted prevalence of CKD in the total population was $6.1 \%$ (95\% CI,4.7-7.6) with $3.5 \%$ presenting with albuminuria $>1 \mathrm{~g} / \mathrm{L}$. CKD prevalence was lower in women (5.7\%) than in men (6.9\%). A linear increase with age was found in CKD prevalence $(\mathrm{P}=0.03)$. In the subgroup of people $>60$ years of age, CKD was diagnosed in 14.3\% (Figure 1). Surprisingly, people living in rural areas showed a similar prevalence of CKD stages $\geq 3$ in comparison to those in urban areas (7.6\% and $5.0 \%$, respectively; $\mathrm{P}=0.08$ ); however, early stages of CKD (1 and 2) were more frequent in urban areas (Figure 2). Before the study, $83 \%$ of people with CKD in rural areas and $62.7 \%$ in urban areas were unaware of their disease.

In the univariate analysis, CKD was significantly associated with hypertension, obesity, physical activity, familial history of kidney disease, and age group (Table 2). After multivariate regression analysis, only age and high blood pressure remained significantly associated with CKD while diabetes, familial history, and obesity failed to reach statistical significance (Table 3 ).

\begin{tabular}{lccc}
\hline Table 2. Association of Chronic kidney Disease With Socio-Demographic and Clinical Factors & \\
\hline & Odds Ratio & 95\% Confidence Interval & P Value \\
\hline Age Group (10 years increment) & 1.08 & $1.02-1.51$ & 0.02 \\
Family History of Renal Disease (yes vs. no) & 1.12 & $0.76-1.45$ & 0.05 \\
School Education (yes vs. no) & 0.99 & $0.76-2.54$ & 0.17 \\
Physical Inactivity (yes vs. no) & 0.65 & $0.57-0.99$ & 0.05 \\
Hypertension (yes vs. no) & 2.15 & $1.61-4.36$ & 0.03 \\
Diabetes (yes vs. no) & 1.22 & $0.33-40.74$ & 0.63 \\
Obesity (yes vs. no) & 1.33 & $1.05-1.98$ & 0.04 \\
\hline
\end{tabular}

${ }^{\mathrm{a}}$ Univariate Analysis.

Table 3. Multivariate Regression Analysis of Factors Associated With Chronic Kidney Disease ${ }^{\mathrm{a}}$

\begin{tabular}{lccc}
\hline & Odds Ratio & 95\% Confidence Interval & PValue \\
\hline Age Group (10 years increment) & 1.03 & $1.00-1.06$ & 0.018 \\
Hypertension (yes vs. no) & 1.12 & $1.02-1.23$ & 0.020 \\
\hline Diabetes (yes vs. no) & 0.80 & $0.25-2.51$ & 0.703 \\
Tobacco Use (yes vs. no) & 0.69 & $0.40-3.81$ & 0.565 \\
Family History of Renal Disease (yes vs no) & 1.43 & $0.38-5.33$ & 0.597 \\
School Education (yes vs. no) & 0.52 & $0.42-12.6$ & 0.722 \\
Obesity (yes vs. no) & 0.77 & $0.24-2.46$ & 0.662 \\
Urban (vs. Rural) Residents & 0.34 & $0.13-0.87$ & 0.024 \\
\hline School Education (yes vs. no) & 0.85 & $0.33-2.19$ & 0.741 \\
\hline
\end{tabular}

${ }^{\mathrm{a}} \mathrm{N}=324$ and Pseudo $\mathrm{R}^{2}=0.106$.

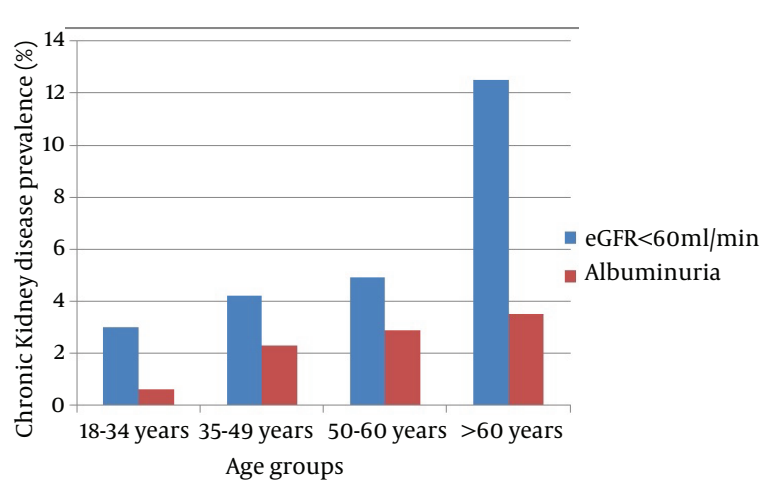

Figure 1. Prevalence of Chronic Kidney Disease According to Age Groups

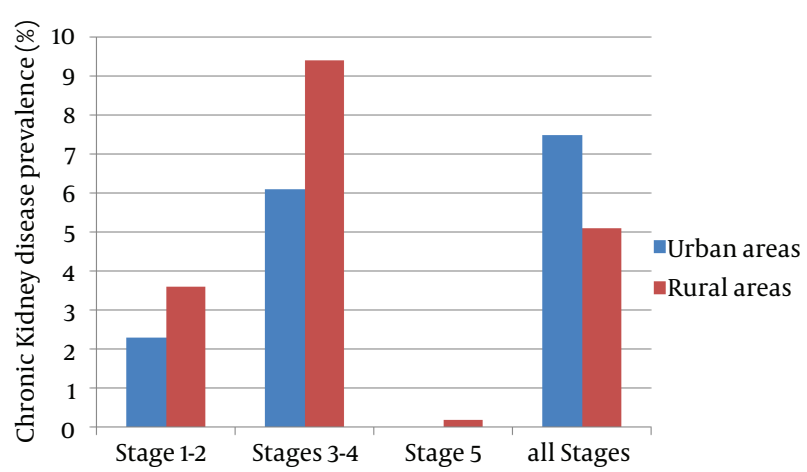

Figure 2. Level of Glomerular Filtration Rate in Urban and Rural Populations 


\section{Discussion}

This was the first study that assessed the burden of CKD, particularly for the early stages ( 1 and 2 ) that are not often seen by specialists at hospital, in a sample of Senegalese population (10). Globally, prevalence of CKD is estimated at $10 \%$ but the rate varies among countries and even within the same countries (11).

In sub-Saharan Africa, estimating the epidemiology of CKD in general population is difficult due to heterogeneity of study populations as well as design and methods used to define CKD (1). Community-based studies are very scarce and most of the previously reported data come from hospital records $(2,3)$. Prevalence of CKD stages $\geq 3$ in our population was lower than previous reports from community-based studies in Ghana (13.2\%) (5) and Kinshasa (12.4\%) (12). This study also confirmed the incremental trend of CKD prevalence with age, which was already described in other populations $(1,3)$. However, urinary abnormalities were more frequently found than the reported prevalence rate of 5.5\% in Iranian school children (13).

As expected, our results found less than one quarter of patients with CKD were aware of their disease, particularly in rural areas where education level was lower and people did not have easy access to healthcare services. Similar low rates of awareness had been commonly reported in other populations (14) and even in health workers (15). More efforts are needed to sensitize governments and populations to the disease burden (1).

CKD risk factors such as high blood pressure, diabetes, obesity, and abdominal obesity $(2,11)$ were frequent among participants but only hypertension was significantly associated with a low GFR. Moreover, age and hypertension independently correlated with CKD. Some risk factors such as infections, genetic predispositions, and environmental or herbal toxins were not evaluated in this study but they might play a prominent role in African populations $(16,17)$. Interestingly, one CKD patient over nine in our study was detected at early stages where therapeutic interventions are most efficient $(2,10)$. In developing countries, the delayed diagnosis of patients at end-stage renal disease (ESRD) has a limited interest because a few proportions of them will not have access to renal replacement therapy $(3,18)$.

In order to achieve long-term reduction in CKD morbidity and mortality, early detection and prevention at population level is the best cost-effective strategy because conservative treatment has limited public health effect $(2,4$, 19). Developed countries spend $2 \%$ to $3 \%$ of their health expenditures in management of patients with ESRD who represent less than $0.03 \%$ of patients (20). The situation is probably worse in low-income countries where healthcare expenditure is often very low and the majority of patients with ESRD die because of dialysis inaccessibility (3). This study has some limitations due to its cross-sectional design; hence, inferences about causality or direction of association should be made with caution. Nevertheless, it gives an insight to the epidemic of CKD stages $\geq 3$ in the northern region of Senegal. More longitudinal studies are urgently needed to yield reliable data about CKD incidence, prevalence, and risk and progression factors in sub-Saharan African populations $(1,3)$. Integrating CKD in other chronic diseases programs would be an interesting approach to increase coverage of screening and awareness in populations. However, mass screening might not be beneficial from a public health perspective because of potential overdiagnosis of cases that might undergo unnecessary investigation or referral to the secondary care, which imposes high cost to the healthcare system (21). In addition, cost-effectiveness of current preventive interventions should be assessed with regard to the local socioeconomic context, particularly in resource-limited areas $(1,4,10)$.

This study reveals a high prevalence of CKD stages $\geq 3$ in northern region of Senegal. The condition in both urban and rural areas is the matter of concern and awareness rate is very low among populations. Early asymptomatic stages are the most frequent ones and associated risk factors are hypertension and aging. Integration of CKD screening into routine general medicine visits would improve early detection and management of patients with renal diseases.

\section{Acknowledgements}

The authors would like to acknowledge the Agence Universitaire de la Francophonie (AUF) for their financial support in this study and Abdoulaye Gueye (archivist) for technical help.

\section{Authors' Contributions}

Sidy Mohamed Seck: study design, data collection, statistical analysis, and manuscript reviewing. Dominique Doupa: study design, data collection, lab analysis, and drafting manuscript. Lamine Gueye: study design and manuscript review. Charles Abdou Dia: data collection and drafting manuscript.

\section{References}

1. Jha V, Garcia-Garcia G, Iseki K, Li Z, Naicker S, Plattner B, et al. Chronic kidney disease: global dimension and perspectives. Lancet. 2013;382(9888):260-72.

2. Couser WG, Remuzzi G, Mendis S, Tonelli M. The contribution of chronic kidney disease to the global burden of major noncommunicable diseases. Kidney Int. 2011;80(12):1258-70.

3. Seck SM. Issues of renal replacement therapy in elders living lowincome african countries. Nephrourol Mon. 2012;4(4):648-9.

4. Hallan SI, Stevens P. Screening for chronic kidney disease: which strategy? J Nephrol. 2010;23(2):147-55.

5. Eastwood JB, Kerry SM, Plange-Rhule J, Micah FB, Antwi S, Boa FG, et al. Assessment of GFR by four methods in adults in Ashanti, Ghana: the need for an eGFR equation for lean African populations. Nephrol Dial Transplant. 2010;25(7):2178-87.

6. Alberti G, Zimmet P, Shaw J, Grundy SM. [cited 2013]; The IDF consensus worldwide definition of the metabolic syndrome. Brussels: International Diabetes Federation. 2006. Available from: http://www. idf.org/webdata/docs/IDF_Meta_def_final.pdf. 
7. Chobanian AV, Bakris GL, Black HR, Cushman WC, Green LA, Izzo JL, Jr, et al. Seventh report of the Joint National Committee on Prevention, Detection, Evaluation, and Treatment of High Blood Pressure. Hypertension. 2003;42(6):1206-52.

8. Levey AS, Bosch JP, Lewis JB, Greene T, Rogers N, Roth D. A more accurate method to estimate glomerular filtration rate from serum creatinine: a new prediction equation. Modification of Diet in Renal Disease Study Group. Ann Intern Med.1999;130(6):461-70.

9. Levey AS, Coresh J, Balk E, Kausz AT, Levin A, Steffes MW, et al. National Kidney Foundation practice guidelines for chronic kidney disease: evaluation, classification, and stratification. Ann Intern Med. 2003;139(2):137-47.

10. Seck SM, Guéye S, Tamba K,, Ba I. Prevalence of chronic cardiovascular and metabolic diseases in Senegalese workers. Prev Chronic Dis. 2010;10.

11. James MT, Hemmelgarn BR, Tonelli M. Early recognition and prevention of chronic kidney disease. Lancet. 2010; 375(9722):1 296-309.

12. Sumaili EK, Krzesinski JM, Cohen EP, Nseka NM. [Epidemiology of chronic kidney disease in the Democratic Republic of Congo: review of cross-sectional studies from Kinshasa, the capital]. Nephrol Ther. 2010;6(4):232-9.

13. Parakh P, Bhatta NK, Mishra OP, Shrestha P, Budhathoki S, Majhi $S$, et al. Urinary screening for detection of renal abnormalities in asymptomatic school children. Nephrourol Mon. 2012;4(3):551-5.

14. Plantinga LC, Boulware LE, Coresh J, Stevens LA, Miller ER, 3rd, Sa- ran R, et al. Patient awareness of chronic kidney disease: trends and predictors. Arch Intern Med. 2008;168(20):2268-75.

15. Minutolo R, De Nicola L, Mazzaglia G, Postorino M, Cricelli C, Mantovani LG, et al. Detection and awareness of moderate to advanced CKD by primary care practitioners: a cross-sectional study from Italy. Am J Kidney Dis. 2008;52(3):444-53.

16. Kanji Z, Powe CE, Wenger JB, Huang C, Ankers E, Sullivan DA, et al. Genetic variation in APOL1 associates with younger age at hemodialysis initiation. J Am Soc Nephrol. 2011;22(11):2091-7.

17. Jha V, Rathi M. Natural medicines causing acute kidney injury. Semin Nephrol. 2008;28(4):416-28.

18. White SL, Chadban SJ, Jan S, Chapman JR, Cass A. How can we achieve global equity in provision of renal replacement therapy? Bull World Health Organ. 2008;86(3):229-37.

19. Black C, Sharma P, Scotland G, McCullough K, McGurn D, Robertson L, et al. Early referral strategies for management of people with markers of renal disease: a systematic review of the evidence of clinical effectiveness, cost-effectiveness and economic analysis. Health Technol Assess. 2010;14(21):1-184.

20. Levey AS, Atkins R, Coresh J, Cohen EP, Collins AJ, Eckardt KU, et al. Chronic kidney disease as a global public health problem: approaches and initiatives - a position statement from Kidney Disease Improving Global Outcomes. Kidney Int. 2007; 72(3):247-59.

21. Taal MW. Screening for chronic kidney disease: preventing harm or harming the healthy? PLoS Med. 2012;9(11). 